This document is the accepted manuscript version of a published work that appeared in final form in Nano letters, copyright @ $\mathrm{American}$ Chemical Society after peer review and technical editing by the publisher.

To access the final edited and published work see: https://dx.doi.org/10.1021/nl504446r

This version has been published under a "All rights reserved" license. 


\title{
Position-Controlled Growth of GaN Nanowires and Nanotubes on Diamond by Molecular Beam Epitaxy
}

\author{
Fabian Schuster, ${ }^{* \dagger}{ }^{\dagger}$ Martin Hetzl, ${ }^{\dagger}$ Saskia Weiszer, ${ }^{\dagger}$ Jose A. Garrido, ${ }^{\dagger}$ María de la Mata, ${ }^{\ddagger}$ Cesar Magen, ${ }^{\text {I }}$ \\ Jordi Arbiol, $, \$, \perp, \|$ and Martin Stutzmann* ${ }^{*}$, \\ †Walter Schottky Institut and Physics Department, Technische Universität München, Am Coulombwall 4, 85748 Garching, Germany \\ "Institut de Ciència de Materials de Barcelona, ICMAB-CSIC, Campus de la UAB, 08193 Bellaterra, Catalonia, Spain \\ "Laboratorio de Microscopías Avanzadas (LMA), Instituto de Nanociencia de Aragon (INA)-ARAID, and Departamento de Física de \\ la Materia Condensada, Universidad de Zaragoza, 50018 Zaragoza, Spain \\ ${ }^{\S}$ Institució Catalana de Recerca i Estudis Avançats, ICREA, 08010 Barcelona, Catalonia, Spain \\ ${ }^{\perp}$ CELLS-ALBA Synchrotron Light Facility, 08290 Cerdanyola, Catalonia, Spain \\ "Institut Català de Nanociència i Nanotecnologia (ICN2), Campus UAB, 08193 Bellaterra, Catalonia, Spain
}

ABSTRACT: In this work the position-controlled growth of $\mathrm{GaN}$ nanowires (NWs) on diamond by means of molecular beam epitaxy is investigated. In terms of growth, diamond can be seen as a model substrate, providing information of systematic relevance also for other substrates. Thin Ti masks are structured by electron beam lithography which allows the fabrication of perfectly homogeneous GaN NW arrays with different diameters and distances. While the wurtzite NWs are found to be Ga-polar, $\mathrm{N}$-polar nucleation leads to the formation of tripod structures
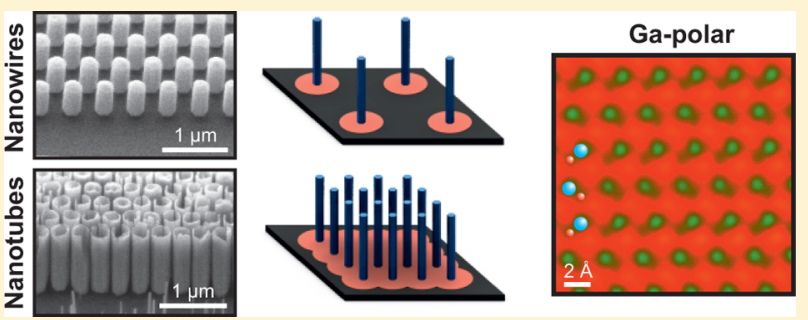
with a zinc-blende core which can be efficiently suppressed above a substrate temperature of $870{ }^{\circ} \mathrm{C}$. A variation of the III/V flux ratio reveals that both axial and radial growth rates are $\mathrm{N}$-limited despite the globally $\mathrm{N}$-rich growth conditions, which is explained by the different diffusion behavior of $\mathrm{Ga}$ and $\mathrm{N}$ atoms. Furthermore, it is shown that the hole arrangement has no effect on the selectivity but can be used to force a transition from nanowire to nanotube growth by employing a highly competitive growth regime.

KEYWORDS: Nanowires, GaN, selective area growth, molecular beam epitaxy, proximity effects, polarity

\begin{abstract}
aN nanowires (NWs) have received wide attention in the
7 recent literature. The process of autocatalyzed GaN NW growth has been realized on various substrates, paving the way to numerous potential applications in the fields of optoelectronics, catalysis, sensing, and photovoltaics. Recently, we have added diamond to the list of applicable substrates, particularly interesting for new routes of UV-emitting optoelectronic devices. ${ }^{1}$ Naturally, the statistical nature of the nucleation process implies problems such as inhomogeneities of the NW dimensions or reproducibility. An obvious solution is the position-controlled or selective area growth (SAG) where position and diameter of the NWs are predefined by a structured substrate. However, this is not only interesting for UV LEDs. The position control of GaN NWs on diamond is strongly desired for quantum computation, i.e., in combination with intentionally implanted, shallow nitrogen-vacancy (NV) centers in diamond where the NWs can simultaneously act as nanocontacts to electrically manipulate the NV charge state (e.g., by forming local $\mathrm{p}$-diamond/n-GaN heterojunctions) and as nanoantennas directly attached to the NV center for optical readout. Additionally, InGaN quantum dots can be embedded in the GaN NWs where the optical resonance can be tuned to match the one of the NV center. With that, the two separately
\end{abstract}

pursued concepts of quantum computation based on solid-state defect centers and quantum dots might be combined to profit from both approaches.

In order to establish GaN NW SAG on diamond substrates, different approaches might be considered. GaN NW SAG was first realized by metalorganic vapor phase epitaxy (MOVPE) with the help of $\mathrm{Si}_{x} \mathrm{~N}_{y}$ masks deposited on a thick, heteroepitaxial $\mathrm{GaN}$ template (short notation: mask/substrate $=\mathrm{Si}_{x} \mathrm{~N}_{y} / \mathrm{GaN}$ ) and later also for $\mathrm{SiO}_{2} /$ sapphire. ${ }^{2,3}$ However, reports on GaN SAG by molecular beam epitaxy (MBE) are few, including attempts of several mask and substrate material combinations: $\mathrm{SiO}_{2} / \mathrm{Si}^{4}{ }^{4} \mathrm{Ti} / \mathrm{GaN}^{,}{ }^{5-7} \mathrm{Si}_{x} \mathrm{~N}_{y} / \mathrm{AlN},{ }^{8,9}$ and $\mathrm{SiO}_{2} /$ AlN. $^{10}$

In this Letter, a systematic study of GaN NW SAG on diamond by prepatterned $\mathrm{Ti}$ masks is presented $(\mathrm{Ti} /$ diamond) where we address the influences of substrate temperature, III/V ratio, growth time, and geometric aspects of NW positioning. In terms of growth, diamond can be seen as a model substrate as it is chemically inert, nonpolar, and not affected by the growth

Received: November 19, 2014

Revised: January 21, 2015 


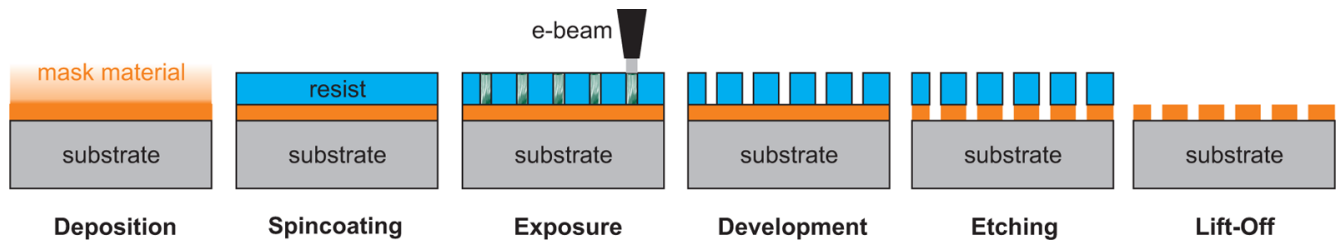

Figure 1. Illustration of the nanomask fabrication process by electron beam lithography. The mask also allows controlled nitrogen implantation to form NV centers in the diamond substrate prior to GaN NW selective area growth. The process details are discussed in the text.
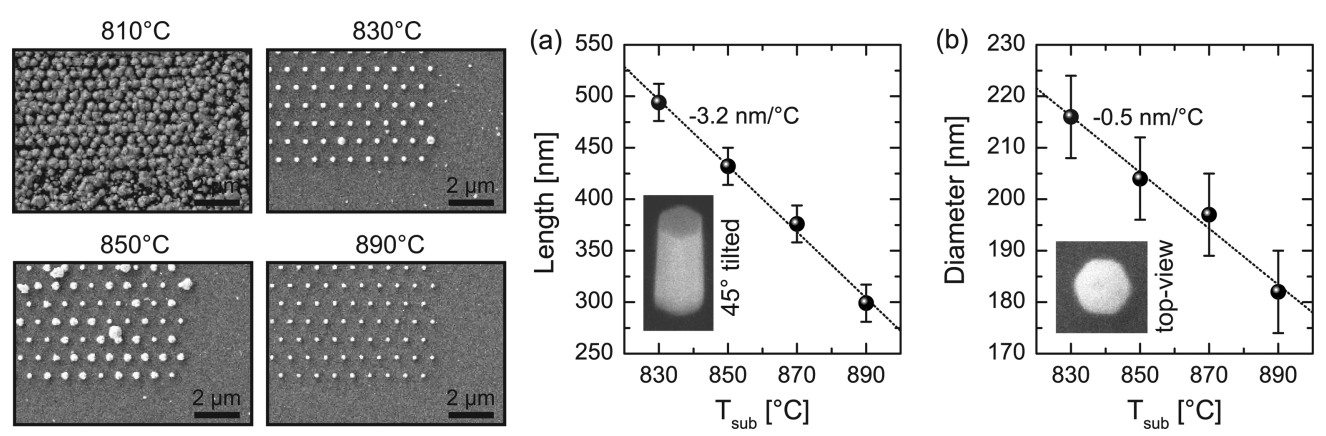

Figure 2. Top-view SEM images of SAG NWs for different substrate temperatures (left). (a) Length and (b) diameter of the NWs for increasing substrate temperature. The series was grown with an $\mathrm{N}$ flux of $0.5 \mathrm{sccm}$ for $90 \mathrm{~min}$.
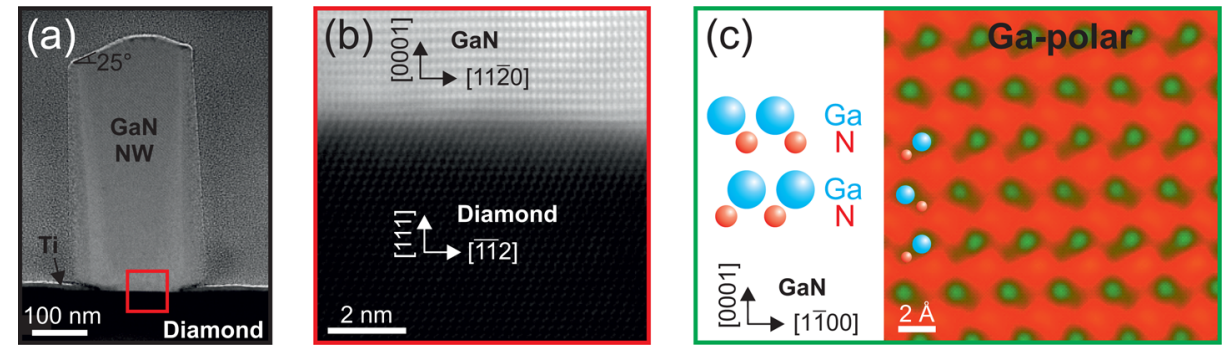

Figure 3. (a) HAADF STEM image of a single GaN NW in the diamond [110] zone axis. The NW clearly extends over the Ti mask hole in lateral direction. (b) The heterointerface shows a smooth and abrupt transition from diamond to wurtzite GaN. (c) Aberration-corrected ABF STEM in the $\mathrm{GaN}[11 \overline{2} 0]$ zone axis reveals the $\mathrm{Ga}-\mathrm{N}$ dumbbells (false colored) with the larger $\mathrm{Ga}$ atoms on top of the characteristic double layers confirming Ga polarity.

conditions, revealing an insight in nucleation and growth undisturbed by the substrate. The model character of this substrate, the abrupt interface, and the absence of interfacial layers have already been successfully used in extracting the impact of the substrate on self-assembled GaN NW growth. ${ }^{1,11}$

A schematic view of the SAG nanomask fabrication process is presented in Figure 1. Diamond (111) plates served as substrates which were coated with a $10 \mathrm{~nm}$ Ti mask. Regular hole arrays were defined by electron beam lithography and subsequently transferred into the $\mathrm{Ti}$ mask by wet chemical etching. A hexagonal arrangement of the holes was chosen which leads to an equal distance to all six next-neighbor holes called period in the following. Hole arrays with periods in the range of 300-5000 $\mathrm{nm}$ and nominal diameters in the range of 40-140 nm were fabricated. More details of the nanomask fabrication process are described in the Methods section.

Influence of the Substrate Temperature. The most important parameter for SAG in general is the substrate temperature. In order to achieve growth on the substrate and suppress it on the mask, differences in Ga sticking coefficients between mask and substrate material are exploited. This defines a temperature window where selective growth can be achieved. In Figure 2 top view scanning electron microscopy (SEM) images of a SAG NW array for different nominal substrate temperatures (thermocouple values) are compared (800 nm period, $100 \mathrm{~nm}$ nominal hole diameter). At $810{ }^{\circ} \mathrm{C}$ nucleation is equally observed on the mask and in the predefined holes, so no selectivity was achieved. By increasing the substrate temperature, the Ga diffusion length on the mask increases, which enhances the probability of $\mathrm{Ga}$ adatoms to reach a mask hole. Consistently, selectivity was achieved for a substrate temperature of $830{ }^{\circ} \mathrm{C}$ which represents the lower limit for successful GaN NW SAG on a Ti mask. The fraction of occupied holes, the so-called yield, is $100 \%$ over the whole sample. An optimal result with homogeneous NWs and a complete suppression of parasitic growth was obtained for 890 ${ }^{\circ} \mathrm{C}$, which is comparable to thermocouple values reported in the literature for homoepitaxial Ti/GaN SAG. ${ }^{5-7}$

Additionally, the length and diameter of the NWs were deduced from SEM images and are plotted in Figures $2 a$ and $b$, respectively. Both axial and radial growth rates decrease for increasing temperature, which can be assigned to increasing $\mathrm{Ga}$ desorption and $\mathrm{GaN}$ decomposition rates.

To evaluate the interface quality and the polarity of the NWs, a cross section was cut by focused ion beam (FIB) normal to the diamond $[\overline{1} 10]$ direction and investigated by aberrationcorrected high-angle annular dark-field (HAADF) scanning transmission electron microscopy (STEM). Figure 3a shows a 

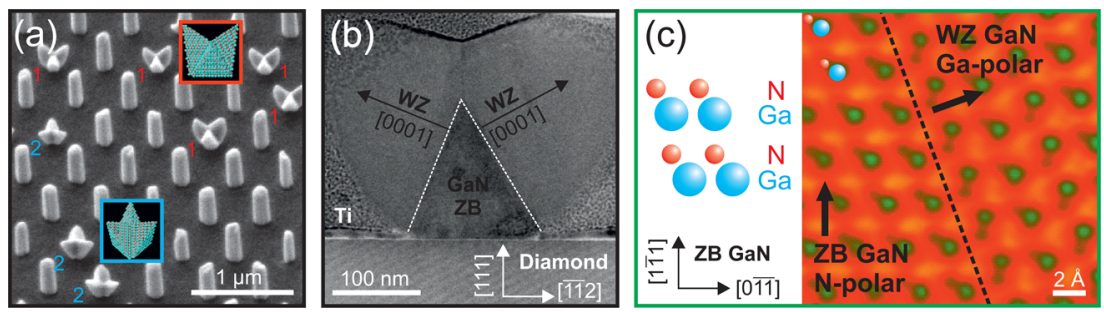

Figure 4. (a) For intermediate growth temperatures tripods nucleate in some holes which show two rotation domains (denoted as 1 and 2). Insets correspond to the 3D atomic models for the tripods in both rotation domains. (b) HAADF STEM image of a tripod cross section in the diamond [110] zone axis consisting of a zinc-blende GaN seed and three wurtzite GaN legs. (c) ABF STEM image of the GaN interface between ZB seed and WZ leg in the GaN [11를 zone axis. The false-colored Ga-N dumbbells indicate an N-polar ZB seed and Ga-polar WZ legs.
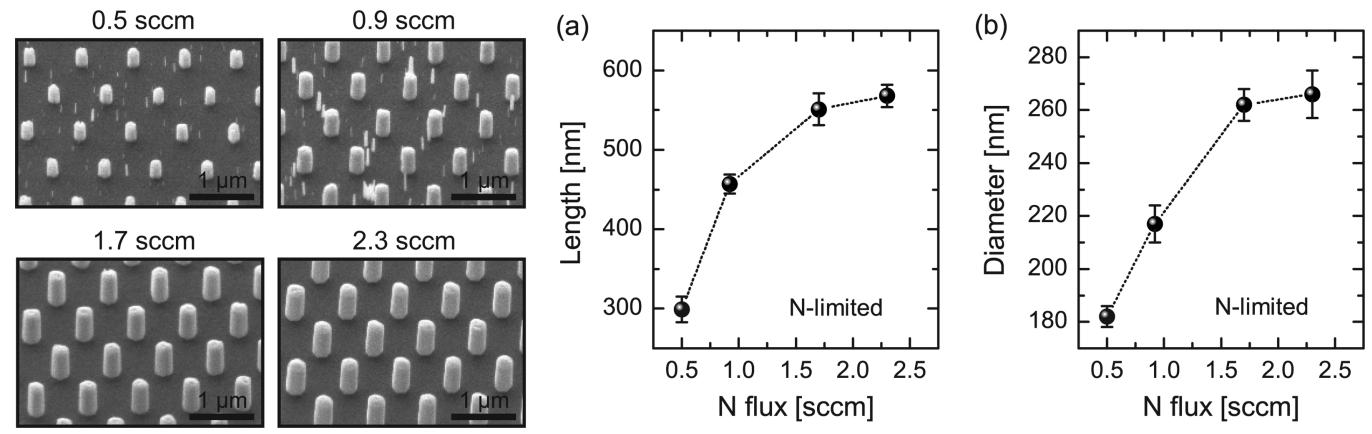

Figure 5. Tilted view SEM images $\left(45^{\circ}\right)$ of SAG NWs for different $\mathrm{N}$ fluxes (left). (a) Length and (b) diameter of the NWs increase with increasing $\mathrm{N}$ flux, indicating an $\mathrm{N}$-limited growth regime. The series was grown with a substrate temperature of $890{ }^{\circ} \mathrm{C}$ for $90 \mathrm{~min}$.

single NW grown in a hole of the Ti mask. The hole diameter is found to be $140 \mathrm{~nm}$, which is substantially broader than the nominal diameter of $100 \mathrm{~nm}$. This is attributed to the wet chemical etching step in the mask fabrication process (Figure 1). The NW top is not exclusively formed by the polar $\{0001\}$ facets but also contains planes of the $\{10 \overline{1} n\}$ family, where an angle of $25^{\circ} \mathrm{C}$ indicates $n=4$ in this particular case (Figure 3a). The NW base region is magnified in Figure $3 b$ and reveals an atomically smooth and abrupt heterointerface. In aberrationcorrected annular bright-field (ABF) STEM imaging the Ga-N dumbbells can be resolved in an atomic resolution to directly determine the polarity of the wurtzite (WZ) GaN in the $[11 \overline{2} 0]$ zone axis. ${ }^{12}$ Figure $3 \mathrm{c}$ shows that the larger $\mathrm{Ga}$ atoms form the top of the characteristic double layers, which corresponds to $\mathrm{Ga}$ polarity as illustrated on the left-hand side. This is in contrast to the $\mathrm{N}$ polarity of spontaneously nucleated GaN NWs on diamond and silicon substrates, ${ }^{1,13}$ which is claimed to be a general requirement for self-assembled NW growth by Fernández-Garrido et al. on all kinds of substrates. ${ }^{14}$ As a consequence, the Ga-polar nucleation seems to be induced by SAG, either by the specific growth conditions or the enhanced diffusion of $\mathrm{Ga}$ on the mask material which leads to a $\mathrm{Ga}$ accumulation in the mask holes.

In the intermediate temperature regime of $830-870{ }^{\circ} \mathrm{C}$, selectivity is observed; however not only NWs nucleate in the mask holes. A different shape of nuclei occurs which we call tripods in the following. In Figure 4a the coexistence of NWs and tripods can be seen for a sample grown at $870{ }^{\circ} \mathrm{C}$ for 90 min with an $\mathrm{N}$ flux of $0.5 \mathrm{sccm}$, where $20 \%$ of the holes are occupied by tripods. In contrast to the nice hexagonal faceting of the wurtzite SAG NWs (Figure 2 insets), the tripods show 3fold symmetry with two rotation domains denoted with 1 and 2 in Figure 4a.

A cross section of a tripod was cut by FIB normal to the diamond $[\overline{1} 10]$ direction. An HAADF STEM image is presented in Figure $4 \mathrm{~b}$ indicating a tetrahedral, zinc-blende (ZB) GaN seed which is bordered by $\{111\}$ planes and has an epitaxial relationship with the diamond substrate of $(\overline{1} 1 \overline{1})$ $[0 \overline{11}]_{\mathrm{GaN}(\mathrm{ZB})} \|(111)[\overline{112}]_{\text {Diamond }}$. Figure $4 \mathrm{c}$ shows an $\mathrm{ABF}$ STEM image of the $\mathrm{ZB} / \mathrm{WZ} \mathrm{GaN}$ interface in the $[11 \overline{2} 0]$ zone axis. The polarity of the $\mathrm{ZB}$ seed is determined to be $\mathrm{N}$-polar, whereas the WZ legs are found to be Ga-polar. Hence, we can conclude that the growth along the Ga-polar direction is favored under the applied growth conditions. While Ga-polar nucleation seeds lead to the formation of NWs, N-polar nucleation seeds induce a transition to $\mathrm{ZB} \mathrm{GaN}$ and the formation of tripods with three Ga-polar WZ GaN legs. A 3D atomic model visualizes the tripod structure correctly for both rotation domains (Figure 4a insets). This result is in agreement with reports on other material systems, showing that an inversion of polarity in the initial seed may induce the formation of nonvertical nanowires ${ }^{15}$ or other complex nanostructures such as tripods and tetrapods. ${ }^{16}$ For substrate temperatures above $870{ }^{\circ} \mathrm{C}$, the tripods disappear completely because of an efficient suppression of the $\mathrm{N}$-polar $\mathrm{ZB}$ nucleation.

Influence of the III/V Ratio. In the following, the optimal substrate temperature of $890{ }^{\circ} \mathrm{C}$ is kept constant, and the $\mathrm{N}$ flux is varied for a given Ga beam equivalent pressure (BEP) of $4.0 \times 10^{-7}$ mbar. This corresponds to a change of the nominal III/V ratio in the range of $0.002-0.014$, which is still in the highly $\mathrm{N}$-rich growth regime. However, these values only give a rough estimation, as they do not account for Ga desorption and $\mathrm{N}$ cracking efficiency. The results are presented in Figure 5 and show an improvement of selectivity for increasing $\mathrm{N}$ fluxes. This is in contrast to the results of Kishino et al., who reported an increase of parasitic growth for increasing $\mathrm{N}$ fluxes on $\mathrm{Ti}$ / $\mathrm{GaN}^{6}$

The impact of the III/V ratio on the NW length is plotted in Figure 5a. The increasing length with increasing $\mathrm{N}$ flux 


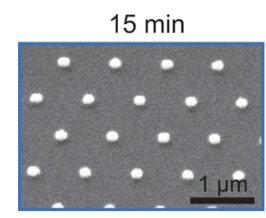

$90 \mathrm{~min}$

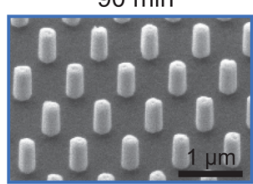

$60 \mathrm{~min}$

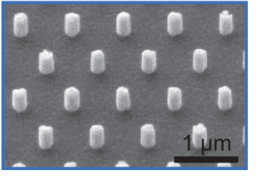

$180 \mathrm{~min}$

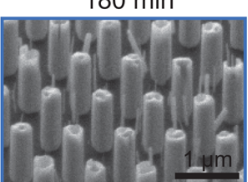

(a)

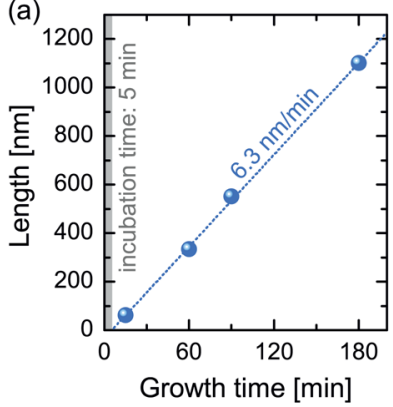

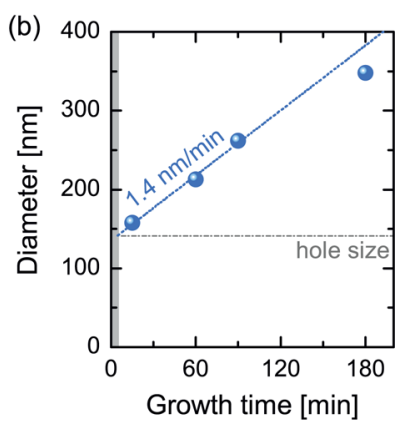

Figure 6. Tilted view SEM images $\left(45^{\circ}\right)$ of SAG NWs for different growth times (left). (a) Length and (b) diameter of the NWs as a function of deposition time, allowing to deduce the indicated axial and radial growth rates. The series was grown with a substrate temperature of $890{ }^{\circ} \mathrm{C}$ and an $\mathrm{N}$ flux of $1.7 \mathrm{sccm}$.
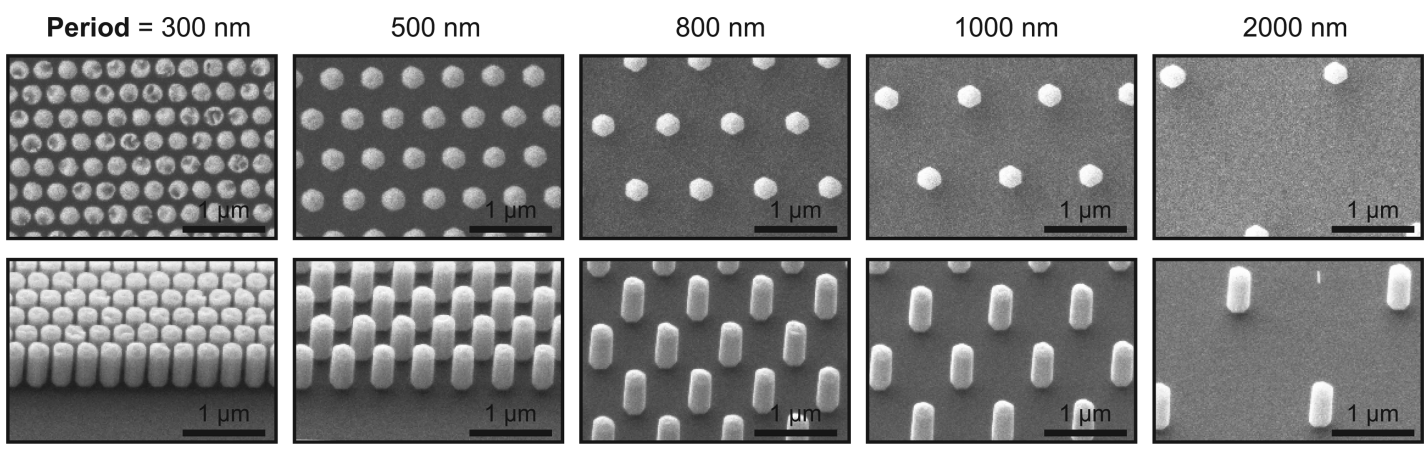

Figure 7. Top view and tilted view SEM images $\left(45^{\circ}\right)$ of SAG NW arrays with different periods. The sample was grown for 90 min at a substrate temperature of $890{ }^{\circ} \mathrm{C}$ and an $\mathrm{N}$ flux of $2.3 \mathrm{sccm}$.

confirms an $\mathrm{N}$-limited growth regime despite the globally $\mathrm{N}$ rich conditions, which at first seems contradictory. In fact, this has also been reported for self-assembled NW growth. ${ }^{11,17,18}$ The reason for this is the different diffusion behavior of $\mathrm{N}$ and Ga. While Ga is mobile on the mask surface and the NW sidewalls within its diffusion length and is therefore also collected from the surrounding area of the NWs, $\mathrm{N}$ radicals are only incorporated where they impinge directly on the growing NW. Otherwise they form $\mathrm{N}_{2}$ molecules which are lost to the growth due to their chemical stability. This leads to locally $\mathrm{N}$ limited conditions for the axial growth on the NW top. Only for the highest applied $\mathrm{N}$ flux a saturation of the length is observed, indicating either the saturation of the plasma cell efficiency or a transition toward Ga-limited conditions on the NW top.

The same applies for radial growth, as the NW diameter presented in Figure $5 \mathrm{~b}$ behaves similar to the NW length. This is in contrast to self-assembled NW growth where the NW diameter is reported to decrease with increasing $\mathrm{N}$ flux. ${ }^{11}$ As the nucleation density increases at the same time, this can be assigned to a competitive effect for Ga. However, for SAG NWs the density is predefined by the mask. Consequently, in the case of SAG both axial and radial growth rates are limited by the amount of impinging $\mathrm{N}$.

Influence of Growth Time. To investigate the nucleation and evolution of SAG NWs over time, the growth time was varied for a fixed $\mathrm{N}$ flux of $1.7 \mathrm{sccm}$. The SEM images are presented in Figure 6. It can be seen that already after 15 min all holes are occupied by homogeneous NW nuclei which proceed to grow in axial and radial directions. After $180 \mathrm{~min}$, the NW top facets look somewhat distorted. Furthermore, parasitic growth on the mask is observed, indicating that there is a finite incubation time for nucleation on the Ti mask. To increase this incubation time the substrate temperature has to be increased which also decreases the axial NW growth rate (Figure 2a). As a consequence, there seems to be a limit of the NW length for maintained suppression of parasitic growth on the mask.

The time evolution of the NW length is presented in Figure 6 a with a deduced axial growth rate of $6.3 \mathrm{~nm} / \mathrm{min}$ for the applied growth conditions. By extrapolation an incubation time of $5 \mathrm{~min}$ is deduced, which is much shorter than for selfassembled GaN NW growth and ensures a homogeneous length of the SAG NWs. ${ }^{1}$ As no variation of the axial growth rate with time is observed, the axial growth is not dependent on the NW length and does not suffer from an increasing shadowing of the substrate by the NWs. A substantial growth rate of $1.4 \mathrm{~nm} / \mathrm{min}$ is observed in radial direction (Figure $6 \mathrm{~b}$ ), which is significantly higher than in self-assembled GaN NW growth. ${ }^{1}$ The reason for this is that the self-regulation of the nucleation density to minimize radial growth is suppressed in SAG. The decrease of the radial growth rate for longer growth times (Figure 6b) indicates that the shadowing effect of the NWs goes at the expense of radial growth. Additionally, the diameter of the nominal $100 \mathrm{~nm}$ mask holes can be deduced by extrapolation to $140 \mathrm{~nm}$ which is in accordance with TEM results (Figure 3a).

Influence of Period and Diameter. Up to now, the influence of the global growth conditions on NW SAG was discussed. However, the local III/V ratio can also depend on the arrangement of the NWs with respect to each other. Therefore, the influence of the mask period on NW SAG is analyzed in the following. Hexagonal hole arrays with periods in the range of $300-5000 \mathrm{~nm}$ were processed on the same substrate and grown under equivalent growth conditions $\left(T_{\text {sub }}\right.$ 
$\left.=890{ }^{\circ} \mathrm{C}, \mathrm{N}_{2}=2.3 \mathrm{sccm}, 90 \mathrm{~min}\right)$. The SEM images of the resulting NW arrays are presented in Figure 7. It can be seen that good selectivity is maintained for all investigated periods. However, for the lowest period of $300 \mathrm{~nm}$, the NW top facets are not homogeneous anymore. This is an indication for a proximity effect in dense NW arrays.

To analyze this effect in more detail, the NW length and diameter are deduced for different periods on two samples grown with $\mathrm{N}$ fluxes of 0.5 and $1.7 \mathrm{sccm}$ and plotted in Figure 8. For both quantities a similar period dependence is found,
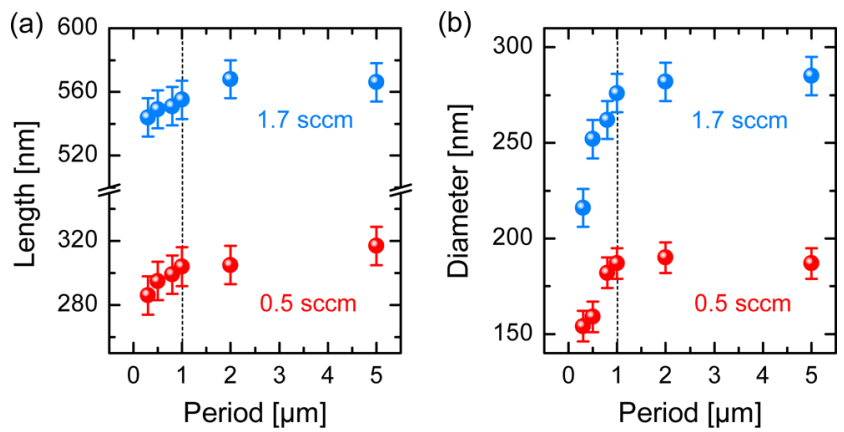

Figure 8. Mask period dependence of the (a) NW length and the (b) NW diameter for two different $\mathrm{N}$ fluxes. Saturation is reached at a threshold period of $1 \mu \mathrm{m}$, which indicates a Ga diffusion length of the order of $500 \mathrm{~nm}$.

namely, a saturation for high periods and a drop for periods smaller than a threshold of approximately $1 \mu \mathrm{m}$. As both growth rates are $\mathrm{N}$-limited according to Figure 5, which is also observed for all periods in Figure 8, a drop of the growth rates due to a competition of the NWs for diffusive Ga adatoms can be ruled out. However, the local III/V ratio also defines the incubation time and the critical nucleus size; thus a perioddependent incubation time affects both length and diameter. Above, the incubation time for a period of $800 \mathrm{~nm}$ at $1.7 \mathrm{sccm}$ $\mathrm{N}$ flux was determined to be $5 \mathrm{~min}$ (Figure 6a). Consequently, by considering the axial growth rate of $6.3 \mathrm{~nm} / \mathrm{min}$, the observed NW lengths for the $300 \mathrm{~nm}$ and $5 \mu \mathrm{m}$ periods correspond to incubation times of 6 and $3 \mathrm{~min}$, respectively. For the diameter, a difference of $43 \mathrm{~nm}$ between the smallest and largest period is already observed after $15 \mathrm{~min}$ of growth (not shown), which confirms different nuclei sizes. However, after $90 \mathrm{~min}$ of growth the difference in diameter is $70 \mathrm{~nm}$, which indicates that the growth rate is also affected. This can be assigned to an increasing shadowing effect of the NW sidewalls from impinging $\mathrm{N}$ radicals and a related drop of the radial growth rate for dense NW arrays.

Consequently, the local III/V ratio mainly influences the nucleation stage, so that the Ga collection area of each mask hole is an important geometric parameter. It can be modeled by a circular area with a radius defined by the Ga diffusion length $l_{\mathrm{Ga}}$ on the Ti mask reduced by the hole area itself. For periods smaller than twice the diffusion length the Ga collection area saturates at the area of its hexagonal parcel, inducing a competition for $\mathrm{Ga}$ adatoms. Hence, the $\mathrm{Ga}$ diffusion length can be estimated as half of the threshold period determined in Figure $8\left(l_{\mathrm{Ga}}=500 \mathrm{~nm}\right)$, which is similar to $400 \mathrm{~nm}$ reported for $\mathrm{SiO}_{2}$ masks. ${ }^{9}$

To realize a highly competitive growth regime, an appropriate mask design with small periods and large diameters is applied, and the resulting NW arrays are compared after a long growth time of $180 \mathrm{~min}$. Exemplary arrays with periods of 300 and $800 \mathrm{~nm}$ and nominal hole diameters of $100 \mathrm{~nm}$ are presented in Figure 9a. While the NWs in the larger period array still look as expected, the formation of hollow nanotubes is observed for the smaller period array. Here, a reduction of the period is equivalent to an increasing overlap of the radial $\mathrm{Ga}$ collection areas on the substrate as illustrated in the middle of Figure 9. For a period of $300 \mathrm{~nm}$ different hole diameters were evaluated. The fraction of holes occupied by NWs or nanotubes is presented in Figure 9c versus Ga collection area, which can be calculated by simple geometric considerations (details in ref 9). A continuous transition from nanowire toward nanotube growth is clearly identified, which reflects a change from $\mathrm{N}$ limited toward Ga-limited growth conditions, induced by simply adjusting the mask design in order to ensure a highly competitive growth regime. To the best of our knowledge, this is the first demonstration of regular SAG GaN nanotube arrays which might have a high potential in applications benefiting from a large surface-to-volume ratio, e.g., catalysis or sensing applications.

Different Substrates. Up to now, all presented results were obtained on single-crystalline diamond substrates with the help of $\mathrm{Ti}$ masks ( $\mathrm{Ti} /$ diamond). An important question is to which extent the growth kinetics depend on the applied mask material on the one hand and the substrate on the other hand. As a proof of concept, the equivalent mask fabrication process was used to prepare silicon (111) $(\mathrm{Ti} / \mathrm{Si})$, sapphire (0001) (Ti/sapphire), and $\mathrm{GaN}$ templates on sapphire with both polarities $(\mathrm{Ti} / \mathrm{GaN})$. For $\mathrm{Ti} / \mathrm{Si}$ the optimized growth conditions $\left(890{ }^{\circ} \mathrm{C}, 1.7 \mathrm{sccm}\right.$, and $90 \mathrm{~min}$ ) also lead to good (a) Period $=800 \mathrm{~nm}$
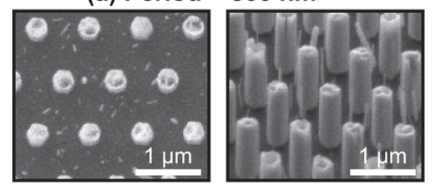

(b) Period $=300 \mathrm{~nm}$

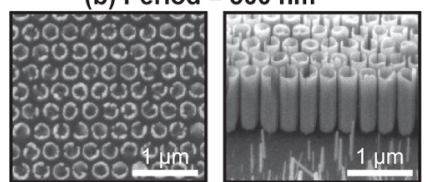

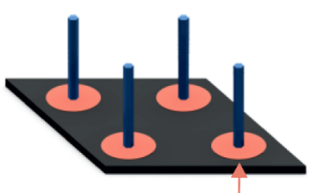

Ga collection

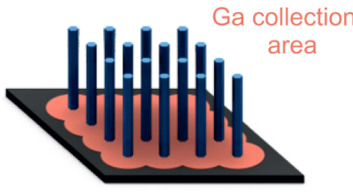

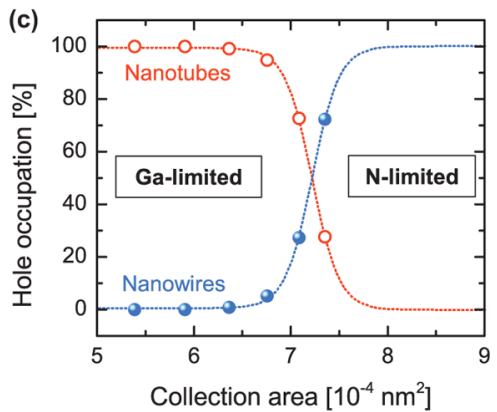

Figure 9. SAG NW arrays with a nominal hole diameter of $100 \mathrm{~nm}$ and periods of (a) $800 \mathrm{~nm}$ and (b) $300 \mathrm{~nm}$. The formation of nanotubes can be assigned to the Ga-limited growth regime due to an overlap of the Ga collection areas. (c) Fraction of mask holes occupied with nanowires and nanotubes versus Ga collection area. The sample was grown for $180 \mathrm{~min}$ at a substrate temperature of $890{ }^{\circ} \mathrm{C}$ and an $\mathrm{N} \mathrm{flux}$ of $1.7 \mathrm{sccm}$. 
(a) Silicon (111)

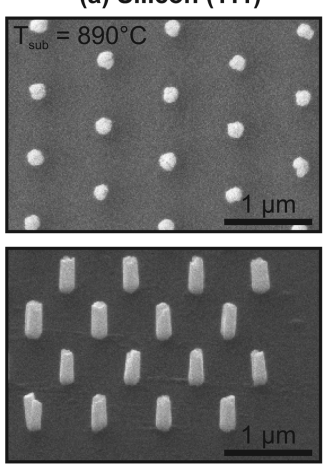

(b) Sapphire (0001)

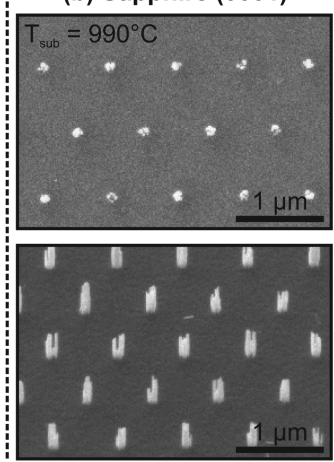

(c) Ga-polar GaN

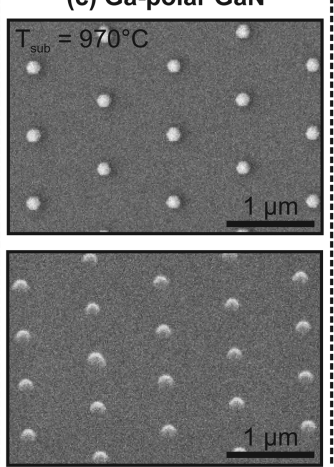

(d) N-polar GaN

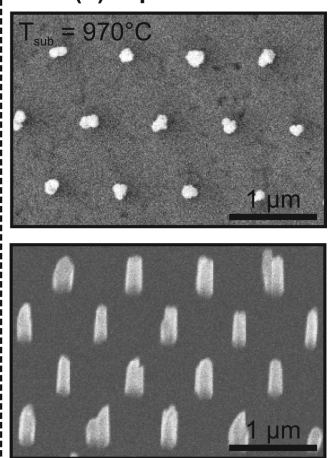

Figure 10. SAG NWs grown on (a) Ti/Si, (b) Ti/sapphire, (c) Ti/Ga-polar GaN, and (d) Ti/N-polar GaN applying the same process as for Ti/ diamond. All samples were grown for $90 \mathrm{~min}$ at an $\mathrm{N}$ flux of $1.7 \mathrm{sccm}$ with indicated substrate temperatures.

selectivity (Figure 10a). This confirms the similar behavior of silicon and diamond substrates in GaN epitaxy, which has also been reported for self-assembled GaN NW growth. ${ }^{11}$ The fact that the SAG NWs are not strictly vertical on silicon might be due to an inhomogeneous $\mathrm{Si}_{x} \mathrm{~N}_{y}$ formation in the mask holes during the incubation time.

For GaN NW SAG on Ti/sapphire the substrate temperatures had to be adapted to $990{ }^{\circ} \mathrm{C}$ due to a different $\mathrm{Ga}$ sticking coefficient in order to achieve selectivity (Figure 10b). This leads to the nucleation of multiple NWs with smaller diameters and different lengths, reflecting a variation in incubation times of the individual NWs.

Finally, GaN NW SAG on GaN templates is investigated $(\mathrm{Ti} / \mathrm{GaN})$ where the NW polarity is defined by the substrate. Both polarities yield proper selectivity at an enhanced substrate temperature of $970{ }^{\circ} \mathrm{C}$ (Figure 10c,d), in agreement with previous reports. $^{5-7}$ However, the elevated temperature obviously favors N-polar NW growth, whereas the Ga-polar nuclei do not show significant vertical growth. This indicates that the polarity in GaN NW SAG is defined by the growth conditions and the substrate, which is in contrast to the irrevocably N-polar growth of self-assembled GaN NWs. ${ }^{14}$

We can conclude that selectivity is achieved by Ti masks on different kinds of substrates. However, an optimization of the growth conditions is required separately for each substrate to improve the NW quality and to avoid effects like multiple NW nucleation in a single hole.

Conclusion. We demonstrated the position-controlled growth of GaN NWs on diamond substrates with the help of structured Ti masks. For a sufficiently high substrate temperature of $890{ }^{\circ} \mathrm{C}$ the growth of highly ordered NW arrays with excellent selectivity was achieved while maintaining $100 \%$ hole occupation. Parasitic growth between the NWs as well as between the SAG arrays was completely suppressed. The heterointerface between diamond and wurtzite $\mathrm{GaN}$ is found to be abrupt and of high quality. For lower substrate temperatures the coexistence of NWs and tripods is observed, which are the result of Ga-polar and N-polar nucleation seeds, respectively.

A variation of the III/V flux ratio revealed an $\mathrm{N}$-limited growth regime for both axial and radial growth rates, where a higher $\mathrm{N}$ flux is preferential in terms of selectivity. The time evolution of the NWs reveals a homogeneous nucleation with a short, period-dependent incubation time as well as a finite incubation time on the Ti mask which defines a maximum NW height while maintaining proper selectivity.
The hole arrangement with different periods and diameters showed no effect on the selectivity, but a geometrically induced transition from a nanowire toward a nanotube growth mode was observed in the highly competitive regime. This was assigned to a change of the local growth conditions from $\mathrm{N}$ limited to Ga-limited. The controlled fabrication of $\mathrm{GaN}$ nanotube arrays will be of interest for applications benefiting from a high surface-to-volume ratio like catalysis or sensing.

Finally, the transfer of GaN NW SAG to other kinds of substrates was demonstrated by applying the equivalent fabrication process and growth conditions with an adapted substrate temperature. We therefore claim our results to be of general relevance for the selective area growth of GaN NWs or nanotubes by using prepatterned substrates.

Methods. Nanomask Fabrication Process. Boron-doped diamond plates (100 ppm) with polished (111) surface orientation served as substrates if not indicated otherwise. The preparation of the nanomasks started with an $\mathrm{O}$ termination of the diamond substrates via oxygen plasma treatment $(200 \mathrm{~W}, 5 \mathrm{~min})$ before the deposition of $10 \mathrm{~nm}$ Ti by thermal evaporation. A low roughness of the mask drastically increases the quality of the SAG. Hole arrays of various diameters $(40-140 \mathrm{~nm})$ and periods $(300-5000 \mathrm{~nm})$ were defined by e-beam lithography using a positive resist (ZEP 520A) and subsequently were transferred into the Ti mask by wet chemical etching in a diluted HF solution (1:1:7 of HF $\left.5 \%-\mathrm{H}_{2} \mathrm{O}_{2} 31 \%-\mathrm{H}_{2} \mathrm{O}\right)$. In contrast to dry etching, this results in a broadening of the hole diameter which was acceptable for this study. After lift-off with n-methyl-2-pyrrolidone (NMP), an additional acetone/isopropanol cleaning procedure, and a second oxygen plasma treatment, the substrates were stored under vacuum. The same fabrication process was applied for the other substrates discussed in Figure 10.

Growth Process. NW growth was performed in a plasmaassisted molecular beam epitaxy (PAMBE) system equipped with an Oxford Applied Research RF plasma source operated at $425 \mathrm{~W}$ and a standard effusion cell for $\mathrm{Ga}(8 \mathrm{~N})$ evaporation with a constant flux of $4.0 \times 10^{-7}$ mbar beam equivalent pressure (BEP). All given substrate temperatures refer to thermocouple values which overestimate the real surface temperature by approximately $100{ }^{\circ} \mathrm{C}$ in the relevant temperature range according to pyrometer calibration with a 2 " silicon wafer. Prior to growth two nitridation steps were performed to transfer the Ti mask into more stable TiN: 10 min at $400{ }^{\circ} \mathrm{C}$ and $5 \mathrm{~min}$ at $800{ }^{\circ} \mathrm{C}$. 
Characterization. The NW arrays were investigated by scanning electron microscopy (SEM) in a Zeiss NVision40. The cross sections of the NW and the tripod were cut by focused ion beam (FIB) normal to the diamond [ 110$]$ direction and investigated by scanning transmission electron microscopy (STEM) in a probe-corrected FEI Titan 60-300 keV microscope operated at $300 \mathrm{keV}$. High-resolution TEM images were obtained in a TECNAI F20 operated at $200 \mathrm{keV}$. 3D atomic models have been created with the Rhodius software. ${ }^{19,20}$

\section{AUTHOR INFORMATION}

\section{Corresponding Authors}

*E-mail: Fabian.Schuster@wsi.tum.de.

*E-mail: Stutz@wsi.tum.de.

\section{Notes}

The authors declare no competing financial interest.

\section{ACKNOWLEDGMENTS}

Financial support from the Deutsche Forschungsgemeinschaft (DFG) via the Forschergruppe 1493, TUM.solar in the frame of the Bavarian Collaborative Research Project "Solar technologies go Hybrid" (SolTec), and the excellence program Nanosystems Initiative Munich is gratefully acknowledged. The authors thank the group of C.E. Nebel at Fraunhofer IAF in Freiburg for supplying the SCD diamond substrates. J.A. acknowledges the funding from the Spanish MINECO MAT2014-51480-ERC (e-ATOM) and Generalitat de Catalunya 2014SGR1638. MdlM thanks the CSIC Jae-Predoc program. The STEM and FIB sample preparation works have been conducted in the "Laboratorio de Microscopias Avanzadas" at "Instituto de Nanociencia de Aragon Universidad de Zaragoza". Authors acknowledge the LMAINA for offering access to their instruments and expertise. Authors also acknowledge ICN2 for the use of their microscopy facilities.

\section{REFERENCES}

(1) Schuster, F.; Furtmayr, F.; Zamani, R.; Magén, C.; Morante, J. R.; Arbiol, J.; Garrido, J. A.; Stutzmann, M. Nano Lett. 2012, 12, 2199.

(2) Hersee, S. D.; Sun, X.; Wang, X. Nano Lett. 2006, 6, 1808.

(3) Bergbauer, W.; Strassburg, M.; Kölper, C.; Linder, N.; Roder, C.; Lähnemann, J.; Trampert, A.; Fündling, S.; Li, S. F.; Wehmann, H.-H.; Waag, A. Nanotechnology 2010, 21, 305201.

(4) Calleja, E.; Ristić, J.; Fernández-Garrido, S.; Cerutti, L.; SánchezGarcía, M. A.; Grandal, J.; Trampert, A.; Jahn, U.; Sánchez, G.; Griol, A.; Sánchez, B. Phys. Status Solidi B 2007, 244, 2816.

(5) Sekiguchi, H.; Kishino, K.; Kikuchi, A. Appl. Phys. Exp. 2008, 1, 124002.

(6) Kishino, K.; Sekiguchi, H.; Kikuchi, A. J. Cryst. Growth 2009, 311, 2063.

(7) Bengoechea-Encabo, A.; Barbagini, F.; Fernandez-Garrido, S.; Grandal, J.; Ristic, J.; Sanchez-Garcia, M.; Calleja, E.; Jahn, U.; Luna, E.; Trampert, A. J. Cryst. Growth 2011, 325, 89.

(8) Bertness, K. A.; Sanders, A. W.; Rourke, D. M.; Harvey, T. E.; Roshko, A.; Schlager, J. B.; Sanford, N. A. Adv. Funct. Mater. 2010, 20, 2911.

(9) Gotschke, T.; Schumann, T.; Limbach, F.; Stoica, T.; Calarco, R. Appl. Phys. Lett. 2011, 98, 103102.

(10) Schumann, T.; Gotschke, T.; Limbach, F.; Stoica, T.; Calarco, R. Nanotechnology 2011, 22, 095603.

(11) Schuster, F.; Weiszer, S.; Hetzl, M.; Winnerl, A.; Garrido, J. A.; Stutzmann, M. J. Appl. Phys. 2014, 116, 054301.

(12) de la Mata, M.; Magen, C.; Gazquez, J.; Utama, M. I. B.; Heiss, M.; Lopatin, S.; Furtmayr, F.; Fernández-Rojas, C. J.; Peng, B.;
Morante, J. R.; Rurali, R.; Eickhoff, M.; Fontcuberta i Morral, A.; Xiong, Q.; Arbiol, J. Nano Lett. 2012, 12, 2579.

(13) Schuster, F.; Laumer, B.; Zamani, R. R.; Magén, C.; Morante, J. R.; Arbiol, J.; Stutzmann, M. ACS Nano 2014, 8, 4376.

(14) Fernández-Garrido, S.; Kong, X.; Gotschke, T.; Calarco, R.; Geelhaar, L.; Trampert, A.; Brandt, O. Nano Lett. 2012, 12, 6119.

(15) Uccelli, E.; Arbiol, J.; Magen, C.; Krogstrup, P.; Russo-Averchi, E.; Heiss, M.; Mugny, G.; Morier-Genoud, F.; Nygard, J.; Morante, J. R.; Fontcuberta i Morral, A. Nano Lett. 2011, 11, 3827.

(16) Utama, M. I. B.; de la Mata, M.; Magen, C.; Arbiol, J.; Xiong, Q. Adv. Funct. Mater. 2013, 23, 1636.

(17) Songmuang, R.; Ben, T.; Daudin, B.; González, D.; Monroy, E. Nanotechnology 2010, 21, 295605.

(18) Fernández-Garrido, S.; Kaganer, V. M.; Sabelfeld, K. K.; Gotschke, T.; Grandal, J.; Calleja, E.; Geelhaar, L.; Brandt, O. Nano Lett. 2013, 13, 3274.

(19) Bernal, S.; Botana, F. J.; Calvino, J. J.; López-Cartes, C.; PérezOmil, J. A.; Rodríguez-Izquierdo, J. M. Ultramicroscopy 1998, 72, 135.

(20) Arbiol, J.; Cirera, A.; Peiró, F.; Cornet, A.; Morante, J. R.; Delgado, J. J.; Calvino, J. J. Appl. Phys. Lett. 2002, 80, 329. 